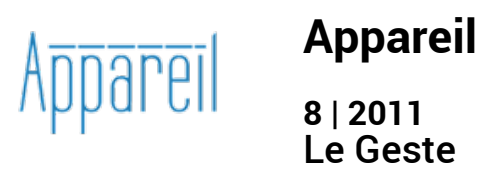

\title{
Le geste
}

Présentation

Philippe Roy

\section{CpenEdition}

Journals

Édition électronique

URL : http://journals.openedition.org/appareil/1354

DOI : 10.4000/appareil.1354

ISSN : 2101-0714

Éditeur

MSH Paris Nord

Référence électronique

Philippe Roy, «Le geste », Appareil [En ligne], 8 | 2011, mis en ligne le 02 novembre 2011, consulté le 22 septembre 2020. URL : http://journals.openedition.org/appareil/1354 ; DOI : https://doi.org/10.4000/ appareil. 1354

Ce document a été généré automatiquement le 22 septembre 2020.

\section{(9) $\odot \Theta \Theta$}

Appareil est mis à disposition selon les termes de la Licence Creative Commons Attribution - Pas d'Utilisation Commerciale - Pas de Modification 4.0 International. 


\section{Le geste}

Présentation

Philippe Roy

1 À voir la diversité des champs ici abordés, un premier constat s'impose : le geste semble être partout. Geste de penser et d'apprendre, geste philosophique ou mathématique, geste politique, socio-économique ou punitif, geste du quotidien ou communicatif, geste technique ou induit par la technologie, geste artistique, cinématographique ou inducteur de création, geste expressif de l'inconscient ou d'énonciation. Quelle est donc la modalité ontologique qui peut rendre compte de cette ligne de variation du geste ? De quelle nature est-il puisqu'il semble être de l'ordre de la pensée, du corps, de l'image, du potentiel ? On trouvera à ce sujet des positions explicites ou implicites dans les articles ici réunis. C'est un premier enjeu de ce numéro consacré au geste: permettre à chaque lecteur d'en questionner l'être ou de se laisser questionner par lui. Il se peut que la question de l'être du geste soit elle-même à questionner, penser le geste est peut-être aussi un geste... vrille du geste. Un deuxième enjeu, qui n'est pas moindre que le premier, est l'analyse d'expériences de gestes ordinaires ou singuliers. Car penser un geste, et on le verra pour chacun des articles, demande toujours de partir de l'expérience (certains posent même le problème du rapport à cette expérience gestuelle, comment la restituer, ne pas la perdre ou la transformer). On ne peut faire face au geste comme on le fait avec un objet. Ce pourquoi les auteurs se sont efforcés de circonscrire les places prises par les gestes qu'ils exposent, de décrire leurs contextes, de déployer parfois leurs topologies, d'esquisser leurs manières d'être ou leur tonalité affective pour délimiter un champ d'expérience. De certains gestes pourront aussi être exposées la genèse, la généalogie, les réactivations, les alliances et mésalliances, les catégories.

2 On ne séparera surtout pas ces deux enjeux car il n'y a pas d'expérience qui ne suscite d'elle-même une question posée par le geste. Inversement, le questionnement sur le geste permet d'en mieux suivre l'allusion en situation. Enfin, partir du geste donne accès à d'autres problèmes dont il serait vain de vouloir écrire ici simplement la liste, demandons-nous seulement si cette entrée par le geste n'induirait pas une 
reconfiguration dans la manière même d'aborder certains problèmes. Encore un tour du geste.

\section{AUTEUR}

\section{PHILIPPE ROY}

Doctorant en philosophie à Paris VIII, Philippe Roy est professeur vacataire en philosophie, chercheur à la MSH Paris Nord (membre de l'équipe du projet « Archives visuelles et sonores sur les migrations africaines clandestines »), co-fondateur du groupe « Gilles Châtelet », intervenant en milieu psychiatrique et régulièrement à la MSH Paris Nord (philippe.roy25@gmail.com). Ses recherches portent sur le concept de geste :- En art, il a publié « Stéphane Blondeau. La naissance est dans le geste » (revue Chimères, $n^{\circ} 60,2006$ ), « Geste cinématographique et cinéma documentaire » (Philosophie \& Cinéma, dir. J.-L. Déotte, L'Harmattan, 2011), est paru en ligne avec vidéo « À propos de "Murmure de peau" d'Isabel Caccia. Une mémoire dans la matière » (2008, http://murmuredepeau.blogspot.com/2008/08/par-philippe-roy.html) et est intervenu à une journée d'études sur Antonin Artaud, « Artaud et le geste » (Paris VII, dir. Évelyne Grossman et Diane Massone, 2011).- En physique et en mathématiques, il a dirigé une formation de formateurs sur le geste en mathématiques (Cafoc de Besançon, 2003) et a élaboré une maquette de logiciel sur l'apprentissage gestuel des mathématiques. Il a donné deux conférences sur le geste et le virtuel au Collège international de philosophie (Tatiana Roque, 2005) et il a publié « Le cri de Gilles Châtelet » (revue Chimères, $n^{\circ} 56,2005$ ). Est à l'étude la publication de son livre L'immeuble du mobile (PUF, collection « MétaphysiqueS », 2012). Il a publié sur le geste dans l'enseignement de la philosophie « Qu'est-ce que s'orienter dans un lycée ?» (L'enseignement de la philosophie est-il émancipateur ?, dir. Laurence Manesse-Césarini, L'Harmattan, 2011)- En politique il a organisé avec Alain Brossat la journée d'études « Se rendre ingouvernables - gestes politiques, résistances de conduite et contre-conduites dans les années 1970 »(MSH Paris Nord, 2011) et est intervenu au colloque Borders, displacement and creation, Université de Porto (2011), « Topolitics versus membrane ». Il a mené un entretien filmé avec Charles Piaget, «Le geste et la palabre » http://www.dailymotion.com/groupechatelet. Est prévue la sortie prochaine de son livre, Trouer la membrane. Penser et vivre la politique par des gestes (L'Harmattan, collection Esthétiques, série Culture \& Politique), et sa thèse porte sur les gestes et diagrammes en politique (dir. Alain Brossat). 